\title{
A VISÃO DOS PESQUISADORES-ORIENTADORES DE UM PROGRAMA DE VOCAÇÃO CIENTÍFICA SOBRE A INICIAÇÃO CIENTÍFICA DE ESTUDANTES DE ENSINO MÉDIO
}

\author{
Researchers-mentors' views about a High School \\ students scientific training
}

\author{
Ana Filipecki ${ }^{1}$ \\ Susana de Sousa Barros ${ }^{2}$ \\ Marcos da Fonseca Elia ${ }^{3}$
}

\begin{abstract}
Resumo: Neste trabalho são apresentadas as opiniões dos pesquisadores-orientadores de um programa de vocação científica sobre a iniciação científica de estudantes de ensino médio. Um questionário on-line foi desenvolvido para esse fim, contendo quatro itens referentes ao perfil do respondente, 15 referentes aos objetivos do Programa, dez relacionados às estratégias de orientação e uma pergunta aberta, na qual o pesquisador expressa sua opinião sobre a I.C.. O questionário foi respondido por 43 pesquisadores (20\%). Os dados referentes aos itens foram analisados em termos de intensidade e consistência das opiniões. $\mathrm{O}$ aspecto sócio-cognitivo do Programa é o que apresenta maior ênfase para os pesquisadores. Os resultados mostram níveis mais elevados de consistência interna na dimensão exeqüibilidade, em ambas as escalas, sugerindo que a visão dos pesquisadores sobre a I.C. expressa-se no fazer. A visão dos pesquisadores-orientadores é que a investigação científica é uma "arte prática" que não se aprende nos livros e sim mediante a imitação e a experiência. Somente num ambiente físico, materializado nos laboratórios e nos eventos científicos é possível desenvolver conhecimentos e habilidades próprios da carreira científica.
\end{abstract}

Palavras-chave: pesquisadores-orientadores. programa de vocação científica. iniciação científica. educação de nível médio.

\begin{abstract}
This paper presents the researchers-mentors opinions' about a scientific vocational program about a scientific researchtraining program for High School students. An on-line questionnaire was developed specifically to this end. The research instrument contains four items referring to the mentor's profile, 15 referring to the Program's goals, 10 related to the mentoring strategies and an open question allowing the researcher to express his/ her opinion about research training for High School students. The questionnaire was answered by 43 researchers (20\%). The data referring to the items were analyzed in terms of intensity and consistency of the opinions. The researchers considered the social-cognitive aspects the most valuable feature of the Program. The results show higher levels of internal consistency in the exeqüibility dimension, in both scales, suggesting that the researchers' views about the Program are expressed in their actions. The mentors" views are that scientific investigation is a "practical art"not directly acquired in the books but through imitation and experience. Only in the proper environment, which is materialized in laboratories and scientific events, it is possible to develop knowledge and habilities inherent to scientific careers.
\end{abstract}

Keywords: researchers-mentors. scientific vocational program. scientific research-training program. high school education.

${ }^{1}$ Mestre em Educação, pesquisadora visitante do Laboratório de Iniciação Científica na Educação Básica, Escola Politécnica da Saúde Joaquim Venâncio/Fiocruz/Rio de Janeiro.E-mail: afilipecki@fiocruz.br

${ }^{2}$ Mestre em Física, professora aposentada do Instituto de Física, Universidade Federal do Rio de Janeiro, UFRJ.

E-mail:susana@if.ufrj.br

${ }^{3}$ Ph.D. em Science Education, professor colaborador do programa de pós-graduação em Informática do IM-NCE/UFRJ.

E-mail:melia@nce.ufrj.br 
Filipecki, A. et al.

\section{Introdução}

A inserção de estudantes de Ensino Médio em ambientes de pesquisa é uma das formas de operacionalizar os programas de iniciação científica (I.C.) que vêm se institucionalizando nos laboratórios das Universidades e Centros de Pesquisa brasileiros. Apesar de sua diversificação no processo de seleção e acompanhamento, esses Programas são balizados pela crença de que a relação pesquisador-orientando contribui para o desenvolvimento pessoal e profissional do jovem.

O propósito deste trabalho é descrever o processo de levantamento das representações dos pesquisadores do Programa de Vocação Científica da Fundação Oswaldo Cruz (Provoc/Rio de Janeiro) sobre os objetivos da I.C. para estudantes de Ensino Médio e as estratégias de orientação que utilizam. O mapeamento utilizou um questionário on-line elaborado para esse fim. Este instrumento foi elaborado com base nas falas dos pesquisadores sobre as práticas de orientação científica em ação, e não por referenciais teóricos e categorias de análise definidas a priori.

Na próxima seção, são descritas as etapas de construção e validação do questionário. Em seguida, são apresentados os resultados e as conclusões.

\section{2. $\mathrm{O}$ instrumento}

Para medir o grau de importância atribuído pelos pesquisadores do Provoc aos objetivos e as estratégias de orientação, optou-se por um questionário fechado. $\mathrm{O}$ instrumento foi desenvolvido em cinco etapas: 1. pesquisa documental; 2. análise de conteúdo; 3. construção e validação da primeira versão do questionário; 4. aplicação-piloto do questionário, e 5. elaboração da versão final.

\subsection{A pesquisa documental e análise de conteúdo}

$\mathrm{Na}$ primeira etapa, foram analisados os documentos pertencentes à Coordenação do Provoc, dando-se ênfase à Ata do Convênio que deu origem ao Programa e ao Número do Jornal Vox Poli (1998), comemorativo do $10^{\circ}$ aniversário do Provoc. Os documentos relacionados ao funcionamento do Programa foram identificados e organizados por meio do mapeamento dos fluxos de processos de trabalho da Coordenação do Provoc/Fiocruz/RJ, utilizando ferramentas da análise estruturada de sistemas (Gane, 1995). Ainda nessa etapa, e visando o resgate da história do Provoc, foi feita uma entrevista não-estruturada com um dos fundadores do Programa, o pesquisador Dr. Luiz Fernando Ferreira. ${ }^{4}$

Os resultados obtidos na pesquisa documental balizaram a formulação de um roteiro de entrevista constituído de quatro grandes eixos: i. os objetivos da I.C.; ii. o trabalho voluntário do orientador; iii. o processo de orientação científica, e iv. a avaliação da orientação científica.

Esse roteiro foi utilizado para entrevistar cinco orientadores da Fiocruz/RJ, das áreas de Filosofia, História, Bioinformática, Patologia e Farmacologia, selecionados com base em

\footnotetext{
${ }^{4}$ Presidente da Fundação Oswaldo Cruz entre maio e junho de 1990 - http://www.fiocruz.br/ingles/
} historia/luisf.htm. 
A visão dos pesquisadores-orientadores...

uma lista sugerida pela Coordenação do Programa, obedecendo aos seguintes critérios: 1 . diversidade epistemológica das áreas de conhecimento dos pesquisadores; 2. experiência acumulada pelo pesquisador na orientação científica; 3. colaboração extensa do pesquisador com o Programa.

As entrevistas, corpus principal de análise de conteúdo, foram gravadas, transcritas integralmente, decompostas em unidades de registro (Bardin, 1977), recortadas, agrupadas e codificadas em torno de temas-eixo (Segenreich, 1990) gerados com base no roteiro de entrevista e das falas dos entrevistados. As categorias temáticas obtidas foram superpostas às categorias identificadas na pesquisa documental, resultando em um esquema constituído por cinco categorias: 1. Objetivos do Provoc (Objet. Provoc); 2. Funcionamento do Provoc (Func. Provoc); 3. Processo de orientação científica (Proc. orient.); 4. Trabalho voluntário (Trab. Volunt.) e 5. História de vida (Hist. vida).

Esse procedimento possibilita identificar relações potenciais de causa e efeito (por exemplo, a relação entre a trajetória profissional do pesquisador e suas estratégias de orientação) e de complementaridade (por exemplo, a posição em relação aos objetivos do Provoc se combina com a estratégia de orientação). As cinco unidades de contexto codificadas deram origem a 275 Unidades de Registro (UR). ${ }^{5} \mathrm{O}$ Quadro 1 apresenta a distribuição das presenças de unidades de registro por participante e por categoria temática. A inclusão de cada UR foi limitada a um máximo de três categorias.

Ainda nessa etapa, e de forma exploratória, foi avaliada a associação entre as catego-

\begin{tabular}{|c|c|c|c|c|c|c|c|}
\hline & \multicolumn{7}{|c|}{ Categoria temática } \\
\cline { 2 - 8 } Participante & UR & Objet. provoc. & Func. provoc. & Proc. orient. & Trab.volunt. & Hist. vida & Total \\
\hline V1 Filosofia & & 1 & 9 & 25 & 4 & 0 & 39 \\
\hline A2 História & 10 & 17 & 10 & 8 & 2 & 47 \\
\hline M3 Patologia & 8 & 15 & 26 & 4 & 13 & 66 \\
\hline C4 Computação & & 10 & 2 & 29 & 8 & 6 & 55 \\
\hline F5 Farmacodinâmica & 4 & 20 & 25 & 14 & 5 & 68 \\
\hline Total & & $\mathbf{3 3}$ & $\mathbf{6 3}$ & $\mathbf{1 1 5}$ & $\mathbf{3 8}$ & $\mathbf{2 6}$ & $\mathbf{2 7 5}$ \\
\hline
\end{tabular}

Quadro 1

Distribuição das presenças de UR por categorias temáticas e participantes ${ }^{6}$

\footnotetext{
${ }^{5}$ Unidade de significação a codificar. O tema, na concepção de Bardin "é a unidade de significação que se liberta naturalmente do texto analisado segundo certos critérios relativos à teoria que serve de guia à leitura (p.105). No caso desse estudo, a leitura do material coletado orientou-se menos por um quadro teórico específico e mais pelas discussões 'instituintes' em torno do Provoc.

${ }^{6} \mathrm{~A}$ identificação do entrevistado utilizou o sistema alfa-numérico. O primeiro dígito identifica o entrevistado, o algarismo a seguir identifica a ordem em que a entrevista foi realizada e os três dígitos seguintes são a abreviatura da área de conhecimento do pesquisador. Por exemplo: A2 his (pesquisador A, segundo entrevistado, da área de História).
} 
Filipecki, A. et al.

rias temáticas e os entrevistados. Foram determinados os valores da variância $\mathrm{x}^{2}(55)$ e do coeficiente de contingência $C(0,41)$. Esses dois valores fornecem o grau de dependência entre as duas variáveis. As discrepâncias entre os valores observados e os esperados ocorreram de forma mais acentuada nas categorias Funcionamento do Provoc e História de vida, nas quais se destacam os pesquisadores das áreas de Bioinformática e de Patologia, respectivamente. As categorias que apresentaram menor discrepância foram Trabalho Voluntário e Objetivos, nessa ordem.

\subsection{Questionário-piloto}

A estrutura do instrumento seguiu, por afinidade temática, o modelo utilizado na pesquisa Case Studies on Lab Work on Five European Countries (Working Paper, 1998) sobre os objetivos do laboratório didático. O primeiro passo foi listar os objetivos originais do Provoc como descritos no Convênio de Fundação do Programa, em 1986:

1. Despertar nos estudantes de $1^{\circ}$ e $2^{\circ}$ graus do CAP o interesse pela pesquisa científica;

2. Distinguir estudantes que se identifiquem com a pesquisa científica;

3. Contribuir para uma escolha profissional correta do estudante;

4. Possibilitar a participação de estudantes no desenvolvimento de tarefas científicas;

5. Oportunizar a vivência do trabalho de laboratório por parte de estudantes, possibilitando-lhes integrar a teoria e a prática;

6. Contribuir para o desenvolvimento das condições pessoais do aluno;

7. Possibilitar a compreensão e permitir a solução de problemas comuns à escola e à empresa;

8. Oferecer condições ao estudante para que, ao final do estágio, apresente relatório crítico das atividades de que participou.

Do conjunto de objetivos mencionados, foram selecionados aqueles diretamente relacionados aos objetivos identificados nas unidades de registro (UR) e na análise documental: (1); (2); (4); (5) e (6). Com exceção dos objetivos (4) e (6), os demais foram adaptados à nomenclatura utilizada pela coordenação do Programa.

Seguindo o modelo de questionário adotado, o instrumento foi dividido em três seções, antecedidas por uma breve descrição do estudo e o convite de participação. A primeira apresentava os 5 objetivos originais selecionados, solicitando-se ao respondente que os ordenasse em função do grau de importância $(1,2,3,4,5)$ : do mais importante (1) ao menos importante (5). Na segunda seção, cada um dos cinco objetivos originais foi relacionado a um conjunto de itens descrevendo as finalidades da IC. Estes foram selecionados das 33 UR pertencentes à categoria $1 \mathrm{da}$ análise de conteúdo (Quadro 1), devendo o pesquisador indicar seu grau de importância em uma escala de 4 opções: muito importante, importante, pouco importante $e$ "não sei". Na terceira seção, cada um dos objetivos foi relacionado a um conjunto de estratégias, selecionadas com base no conjunto das 115 UR pertencentes à categoria 3 da análise de conteúdo (Quadro 1), que poderiam ser utilizadas para alcançar o objetivo indicado. Nessa 
A visão dos pesquisadores-orientadores...

seção, o pesquisador indica o grau de utilidade de cada estratégia na seguinte escala: muito útil, útil, pouco útil, e mais uma quarta opção - "não se aplica".

Para validação do questionário quanto à abrangência, pertinência e adequação, este foi submetido à apreciação de 4 juízes, especialistas em educação em ciências. Na elaboração da versão final, foram acatadas três modificações sugeridas por esse painel: i. eliminar a primeira seção e juntar os objetivos da IC, isto é, os originais com aqueles identificados nas análises de conteúdo e documental; ii. introduzir uma escala de exeqüibilidade (muito factível, factivel, pouco factivel e não se aplica), relacionando o grau de importância de cada objetivo e estratégia ao nível de operacionalização e iii. diminuir o número de itens dos 81 originais $(30$ relacionados aos objetivos e 51 às estratégias) para 54 (23 relativos aos objetivos e 31 às estratégias).

A versão corrigida foi avaliada por quatro pesquisadores orientadores de programas congêneres. Nessa oportunidade, dois pesquisadores apontaram o papel da escola como espaço de realização da I.C. que seria desenvolvido pelo professor do Ensino Médio em colaboração com o pesquisador na orientação dos estudantes. Dessa forma, na versão final foi introduzida uma terceira seção voltada para a identificação dos possíveis espaços de realização da I.C.

\subsection{O questionário final}

Tendo em vista o número elevado de orientadores registrados no banco de dados do Provoc (253) no período entre 1987-2003, optou-se por uma aplicação on-line do questionário. Em colaboração com o Núcleo de Computação Eletrônica da Universidade Federal do Rio de Janeiro (NCE/UFRJ), o instrumento foi revisto e adaptado para essa modalidade, ${ }^{7}$ gerando a versão final, constituída de quatro itens referentes ao perfil do respondente, 15 itens relacionados aos Objetivos do Programa (oito originais e sete complementares) e dez itens relacionados às Estratégias de orientação. Os 25 itens referentes aos objetivos e as estratégias foram construídos segundo uma escala de intensidade de Likert de três opções (muito, \pm , pouco) sobre o respectivo grau de importância e de exeqüibilidade, com mais uma opção alternativa "não se aplica". Uma pergunta aberta sobre o espaço de realização da I.C. para estudantes de Ensino Médio foi introduzida no final do questionário (Figura 1). O julgamento concomitante da importância e da exeqüibilidade do item permite discriminar o que é desejável e que deveria ser perseguido, pelo Programa, dos aspectos que realmente poderiam ser atingidos, na visão dos orientadores.

\footnotetext{
${ }^{7} \mathrm{O}$ critério de delimitação do número de itens visou a obtenção otimizada dos dados sem comprometer a fidedignidade do instrumento (Kerlinger, 1980, p. 156).
} 
Filipecki, A. et al.

\section{Seção 1}

\section{OBJETIVOS DO PROGRAMA DE VOCAÇÃO CIENTÍFICA (PROVOC)}

Nesta seção apresentamos um conjunto de objetivos da iniciação científica.

Por favor, pense sobre sua relevância e possibilidade de realização.

\begin{tabular}{|c|c|c|c|c|c|c|c|c|c|}
\hline \multicolumn{2}{|r|}{ Objetivos } & \multicolumn{4}{|c|}{ Importância } & \multicolumn{4}{|c|}{ Exeqüibilidade } \\
\hline & O objetivo do Programa é & MImp & $\operatorname{Imp}$ & PImp & NApI & MFac & Fac & PFac & NApl \\
\hline $\begin{array}{l}1 \\
.\end{array}$ & $\begin{array}{l}\text { despertar nos estudantes } \\
\text { do Ensino Médio o } \\
\text { interesse pela pesquisa } \\
\text { científica. }\end{array}$ & O & $\mathrm{O}$ & $\mathrm{O}$ & $\mathrm{O}$ & O & $\mathrm{O}$ & 0 & $\mathrm{O}$ \\
\hline 15 & $\ldots$ & 0 & $\mathrm{O}$ & $\mathrm{O}$ & 0 & 0 & 0 & 0 & 0 \\
\hline
\end{tabular}

Seção 2

ESTRATÉGIAS DE ORIENTAÇÃO CIENTÍFICA

Nesta Seção apresentamos algumas estratégias que solicitamos sejam julgadas de acordo com sua importância e possibilidade de realização. Sabemos que existem diferentes estratégias de orientação científica e que essas são freqüentemente combinadas ou misturadas. Mesmo assim, tente manter o foco das idéias nas situações descritas.

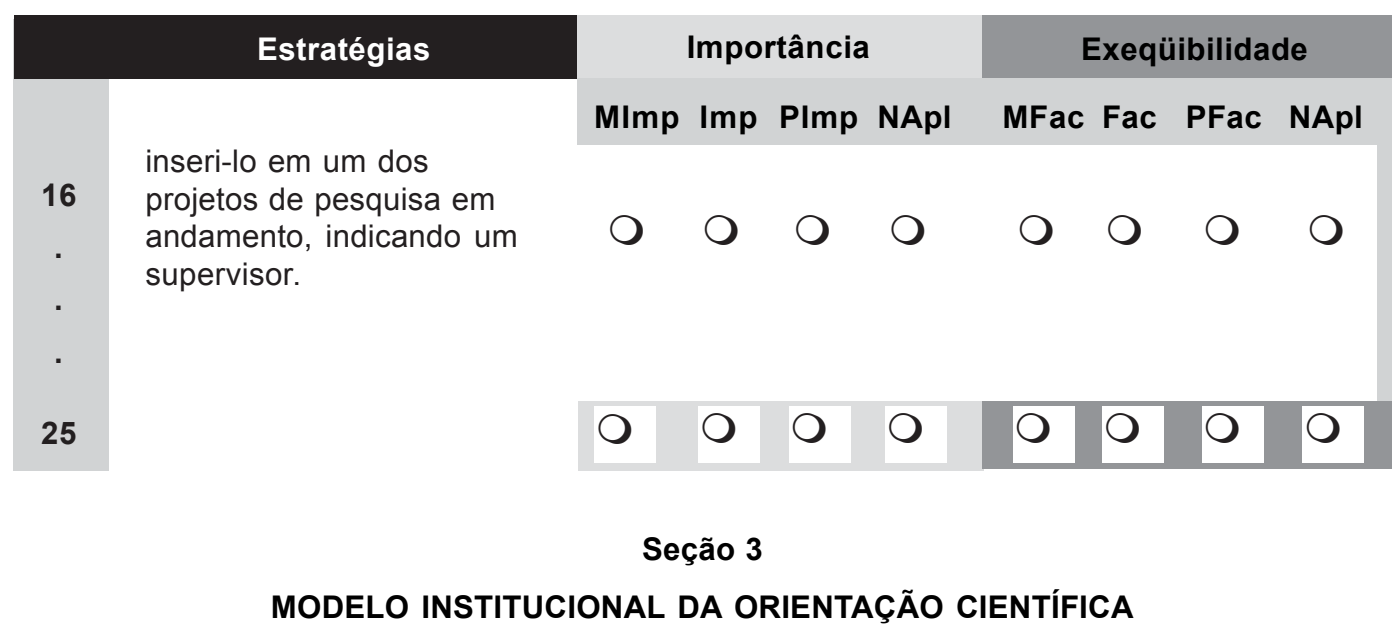

Nesta seção solicitamos sua opinião sobre as instituições que podem, a princípio, realizar programas de iniciação cientítica para estudantes de ensino médio. A iniciação científica de estudantes de ensino médio é uma atividade restrita aos centros de pesquisa e universidades?

MImp-muito importante; Imp -importante; PImp - pouco importante; NApl - não se aplica;

MFac-muito factível; Fac- factível; PFac- pouco factível

Figura 1

Excerto do questionário 
A visão dos pesquisadores-orientadores...

\section{Resultados}

Os 217 pesquisadores com e-mails registrados na Coordenação do Programa foram convidados a participar da pesquisa, preenchendo o instrumento disponibilizado no endereço eletrônico http://146.164.248.51/provoc/wcprovoc.asp. O questionário foi respondido por 43 pesquisadores (20\%), no período entre abril e julho de 2004, incluindo cinco respostas de um conjunto de dez orientadores atualmente pertencentes a outras instituições de pesquisa. $O$ Quadro 2 apresenta a distribuição relativa dos respondentes por cada unidade da Fiocruz/RJ.

\begin{tabular}{|l|c|c|c|}
\hline Instituto & Enviado & Recebido & Percentual*(\%) \\
\hline Instituto de Tecnologia de Imunobiológicos & 4 & 0 & 0 \\
\hline Centro de Informação Científica e Tecnológica & 2 & 0 & 0 \\
\hline Casa de Oswaldo Cruz & 19 & 3 & 16 \\
\hline Escola Nacional de Saúde Pública & 19 & 3 & 16 \\
\hline Escola Politécnica Joaquim Venâncio & 3 & 2 & 67 \\
\hline Instituto de Tecnologia de Fármacos & 13 & 3 & 23 \\
\hline Instituto Fernandes Figueira & 7 & 1 & 14 \\
\hline Instituto Nacional de Controle e Qualidade em Saúde & 9 & 3 & 33 \\
\hline Instituto Oswaldo Cruz & 121 & 20 & 17 \\
\hline Instituto de Pesquisa Evandro Chagas & 4 & 1 & 25 \\
\hline Presidência & 6 & 2 & 33 \\
\hline Outras & 10 & 5 & 50 \\
\hline Total & 217 & 43 & \\
\hline
\end{tabular}

* - Recebido/enviado

\section{Quadro 2}

Distribuição dos respondentes por unidade da Fiocruz/RJ8

\subsection{Análise dos dados}

\section{Objetivos I.C.}

Os 15 itens do questionário referentes aos objetivos foram classificados em dois grupos. O primeiro, contendo oito itens, é constituído pelos objetivos originais do Programa de Vocação Científica. O segundo grupo, denominado complementar, é composto de sete itens

\footnotetext{
${ }^{8}$ Todos os respondentes têm mais de cinco anos de experiência.
} 
Filipecki, A. et al.

extraídos da pesquisa documental e da análise de conteúdo. A consistência interna de cada uma dessas escalas, medida pelo coeficiente estandardizado de Cronbach, ${ }^{9}$ é apresentada na Tabela 1 separadamente para o grau de importância e de exeqüibilidade dos itens. Os valores entre parênteses nas subescalas original e complementar indicam os valores normalizados para 15 itens. Os dados indicam que os itens podem ser considerados como componentes de um constructo e subconstructos, aqui denominados "Objetivos I.C.", sendo ligeiramente mais consistentes no aspecto da importância.

Tabela 1

Consistência interna das escalas relacionadas aos objetivos IC*

\begin{tabular}{lcc}
\hline & \multicolumn{2}{c}{ Escala } \\
\cline { 2 - 3 } & Importância (\%) & Exeqüibilidade (\%) \\
\hline Objetivo original (8 itens) & $74(84)$ & $84(91)$ \\
Objetivo complementar (7 itens) & $68(82)$ & $72(85)$ \\
Objetivo geral (15 itens) & 83 & 88 \\
\hline
\end{tabular}

* Os valores entre parênteses foram normalizados para 10 itens pela fórmula KR21.

Analisando-se a freqüência das escolhas por grupo, observa-se que os respondentes consideram, de modo geral, os objetivos Mimp ou Imp: originais (89\%) e complementares (86\%). Individualmente, os percentuais Mimp e Imp relacionados aos objetivos originais são superiores a $80 \%$, com exceção do item 13, "contribuir para o desenvolvimento das condições pessoais do aluno", que corresponde a $77 \%$. Os itens 2 e 5 dos objetivos adicionais relacionados à carreira científica fogem a esse padrão: "formar pesquisadores o mais precocemente possivel" (70\%) e "incentivar o estudante a seguir a carreira cientifica" (77\%).

Quanto à exeqüibilidade, os objetivos também são considerados MFac e Fac: originais $(87 \%)$ e complementares (83\%). Dentre os objetivos originais, "desenvolver no estudante a habilidade de solucionar problemas" - é o que apresenta menor percentual de exeqüibilidade (74\%). Dos objetivos complementares, o menos exeqǘvel (67\%) é "formar pesquisadores o mais precocemente possivel'.

\section{Pontuação média dos itens da escala Objetivos}

Utilizando uma escala intervalar de três pontos $(\mathrm{MImp}=3$; $\operatorname{Imp}=2 ; \mathrm{PImp}=1)$ e $(\mathrm{MFac}=3 ; \mathrm{Fac}=2 ; \mathrm{PFac}=1)$, foi determinado o grau médio de importância e de exeqüibilidade para cada item individualmente (Figura 2). Observa-se, pelo gráfico da Figura 2, que os pesquisadores são mais cautelosos em relação à sua exeqüibilidade.

${ }^{9}$ http://www.ats.ucla.edu/stat/spss/faq/alpha.html 
A visão dos pesquisadores-orientadores...

Nesta escala, o objetivo que apresenta maior desvio percentual entre importância e exeqüibilidade é "desenvolver no estudante a habilidade de integrar teoria e prática" (18\%), competência-chave da iniciação científica. O item 10 - "permitir que o estudante conheça como os cientistas trabalham profissionalmente" - tem desvio percentual nulo. Os objetivos que apresentam menor escore de importância e exeqüibilidade, conforme discutido anteriormente, são aqueles relacionados à carreira científica (itens 2 e 5): "formar pesquisadores o mais precocemente possivel" e "incentivar os estudantes a seguir a carreira cientifica". O item 1 - "despertar nos estudantes do Ensino Médio o interesse pela pesquisa científica - é o objetivo que tem mais consenso entre os pesquisadores.

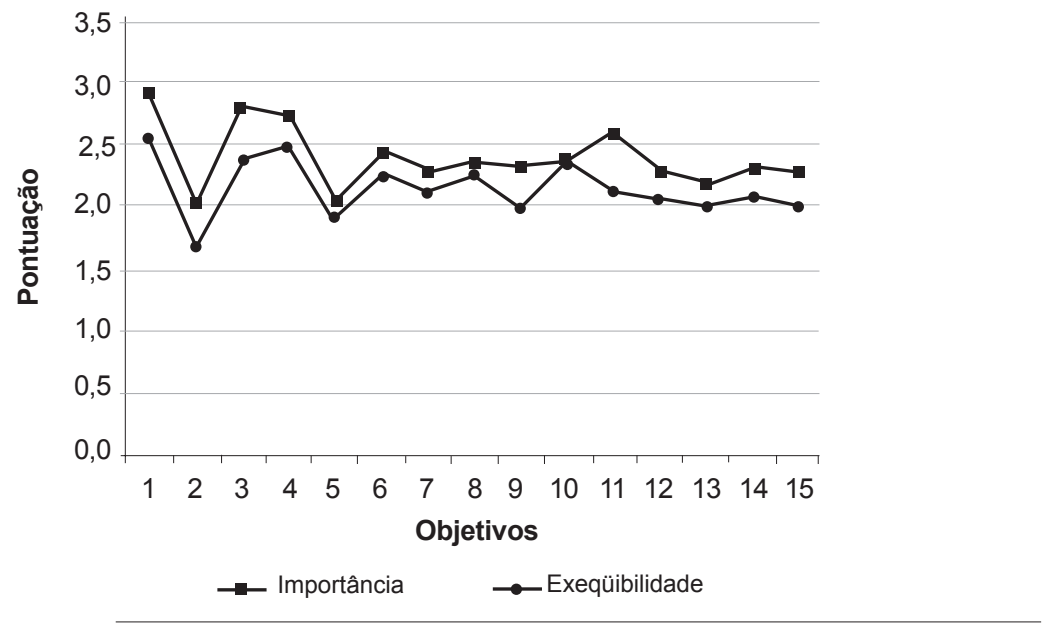

Figura 2

Pontuação média dos objetivos

\section{Estratégias IC}

Os dez itens do questionário que se referem às estratégias de orientação foram extraídos da análise de conteúdo e classificados em dois grupos, comportamental (quatro itens) e procedural (seis itens): o primeiro, envolvendo aspectos da formação geral do orientando, e o segundo, aspectos da área de conhecimento e estilo de orientação do pesquisador. A Tabela 2 e a Figura 3 resumem, para as subescalas referentes às estratégias, análises análogas às que foram apresentadas anteriormente para os objetivos.

As estratégias também são consideradas MImp ou Imp - procedural (86\%) e comportamental (91\%) - e Mfac e Fac - procedural (78\%) e comportamental (87\%). Individualmente, a exeqüibilidade (MFac e Fac) das estratégias procedurais é superior a 95\%, com exceção dos itens "apresentar textos relacionados às pesquisas para que o estudante proponha um projeto" (58\%) e "treinar o estudante na utilização dos equipamentos e manipulação de amostras" (77\%). Quanto às estratégias comportamentais, foge ao padrão "fornecer modelos que possam auxiliá-lo no seu desempenho profissional futuro" (77\%). 
Filipecki, A. et al.

Tabela 2

Consistência interna das escalas relacionadas às estratégias IC*

\begin{tabular}{lcc}
\hline & \multicolumn{2}{c}{ Escala } \\
\cline { 2 - 3 } & Importância (\%) & Exeqüibilidade (\%) \\
\hline Estratégia comportamental (4 itens) & $0,47(69)$ & $0,70(85)$ \\
Estratégia procedural (6 itens) & $0,52(64)$ & $0,76(84)$ \\
Estratégia geral (10 itens) & 66 & 85 \\
\hline
\end{tabular}

* Os valores entre parênteses foram normalizados para 10 itens pela fórmula KR21.

\section{Pontuação média dos itens da escala Estratégias}

Os pesquisadores demonstram, também, para as estratégias, graus de expectativa de realização inferiores aos graus de importância atribuídos, ou seja, são mais cautelosos, embora essas diferenças sejam menos acentuadas nas estratégias. Duas destas são praticamente tão importantes quanto factíveis (desvio de 3\%): "treinar o estudante na utilização dos equipamentos e manipulação de amostras" e "fornecer modelos que possam auxiliá-lo no seu desempenho profissional futuro".

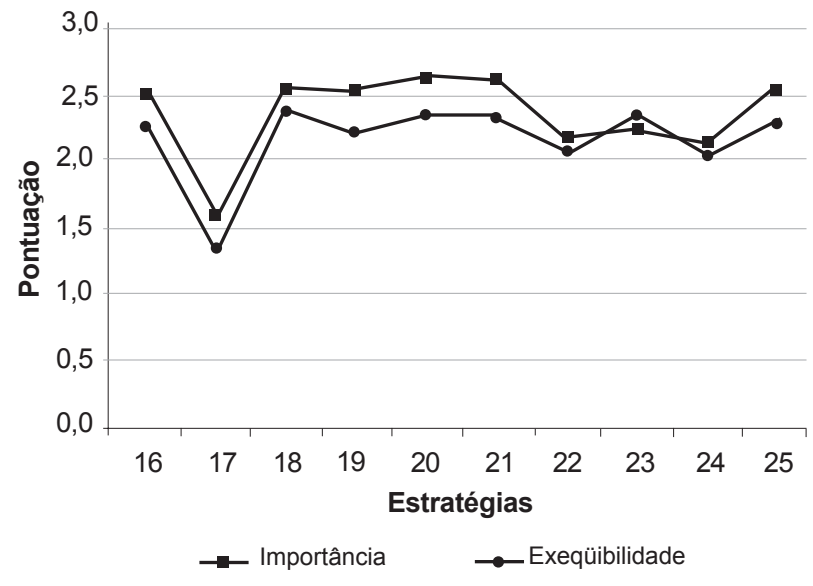

\section{Figura 3}

Pontuação média das estratégias 
Modelo institucional I.C.

Quanto às respostas dos pesquisadores para a pergunta: "A IC de estudantes de Ensino Médio é uma atividade restrita aos centros de pesquisa e universidades?", 56\% afirmaram que não. Nesse grupo, 20\% apontam as instituições de Ensino Médio e as escolas técnicas como espaços que poderiam realizar a I.C., 40\% indicaram instituições privadas (empresas e laboratórios), e 10\% escolas e empresas.

Os excertos apresentados a seguir ilustram a opinião dos pesquisadores:

P1. (...) tenho notado que os centros de pesquisa não têm fornecido aos jovens alunos a possibilidade de vislumbrar um horizonte mais amplo do que aquele do laboratório em que ele se inseriu, levando-o muito mais a agir como técnico do que incentivá-lo a pensar. Pensamento este que o fará questionar a sua escolha e tomar a decisão de seguir ou não o caminho da atividade cientifica.

P2. Acredito que a manutenção do programa em centros de excelência e instituições onde a prática cientifica seja a força motriz que as movimenta é de extrema importância para a identidade e finalidade do programa. Entretanto, outros programas poderiam ser criados com uma roupagem diferente, talvez tecnológica, que se encarregaria de apresentar outras instituicões e outras atividades, além da cientifica, aos alunos, mas, na minha visão, nestes lugares a figura do "orientador" tem que ser muito bem trabalhada, pois ao contrário das universidades e Institutos de pesquisa, como a Fiocruz, muitas vezes nos centros tecnológicos não há formação, desta forma os profissionais podem ter dificuldades em realizar a orientação e eventualmente alcançar os objetivos do programa.

P3. Em função das condições da maioria dos centros de ensino no Brasil, esta possivelmente é a situação atual. Entretanto, a possibilidade de desenvolver projetos de pesquisa no nivel da Iniciação Científica deveria ser oferecida também em escolas do segundo grau. Com a carência das escolas, este programa no momento só e factivel em centros de pesquisa ou universidades.

P4. Acredito que podemos desenvolver o programa de iniciação cientifica em outras instituiçoes, como escolas, empresas produtivas, secretarias de saúde, etc.

P5. O aluno não precisa estar em um centro de pesquisa para realizar este tipo de atividade. Nesse sentido, a participação do orientador é muito importante no sentido de vislumbrar possibilidades de estudos em vários tipos de ambiente.

P6. Do meu conbecimento, além dos Institutos de Pesquisa do País, creio que somente as Universidades públicas brasileiras apresentam alguma condição adequada para ministrar esse tipo de treinamento.

P7. Creio que o que falta ao PROVOC é a implantação, ainda na escola, de um despertar inicial onde o aluno pudesse, em seu ambiente escolar, participar de alguma atividade de pesquisa compativel com o seu nivel escolar. 
Filipecki, A. et al.

P8. O aluno do PROVOC que vem para um Laboratório da FIOCRUZ requer uma adaptação à realidade da pesquisa, da qual ele não tem a menor noção, e isso requer que o pesquisador dedique pacientemente o seu tempo já ocupado pelos numerosos compromissos inerentes à realidade da pesquisa. Creio que o aluno já devesse vir com uma preparação inicial da sua escola. Depende de cada pesquisador a decisão de investir seu tempo na formação de um aluno que freqüentemente não fica no laboratório após o PROVOC para dar um retorno daquilo que the foi ensinado.

P9. Acho que não é uma exclusividade dos centros de pesquisa ou universidades. O ideal seria que as escolas pudessem prover essa visão ao aluno, com laboratórios e gente preparada para o ensino da ciência.

P10. Sim, sendo a pesquisa no Brasil, exceto honrosas exceções, desenvolvida somente em Universidades Públicas e Institutos de Pesquisas, não cabe sugerir nenbum outro local. Entretanto, não bá impedimento que se procure faz̧er investigações (experimentos) cientificas nos locais de ensino do curso médio.

P11. As instituições mais apropriadas a realizarem programas de iniciação cientifica para estudantes são as universidades públicas, por sua tradição nas questões de ensino. Instituições de pesquisa como a Fiocruz podem contribuir, mas o ambiente acadêmico e o convívio com estudantes universitários podem auxiliar na escolha da carreira do aluno.

Nessas opiniões, observa-se uma diversidade de aspectos relacionados à I.C. Destaca-se a importância atribuída pelo pesquisador à qualidade da orientação. A "boa" orientação incentiva o estudante a pensar e evita o trabalho repetitivo e pouco criativo. Além disso, um "bom" orientador sabe vislumbrar oportunidades de I.C. em diferentes ambientes. A relação da I.C. com a escolha da carreira é outro aspecto abordado. Nesse sentido, a ambiência é o elemento em destaque. Foram também mencionadas questões relativas ao papel da escola e a ausência de ações que preparem o estudante previamente à sua participação em Programas dessa natureza. Observa-se, ainda, o reconhecimento, pelos pesquisadores, da precarização das escolas de Ensino Médio: falta de recursos humanos preparados para o ensino de ciências, falta de recursos materiais, e a necessidade de mudança.

\section{Discussão dos resultados}

Esta seção está dividida em duas partes. Na primeira, são retomados os principais aspectos relacionados à qualidade do instrumento utilizado, dando-se ênfase às diferenças nas correlações entre as escalas e suas dimensões. $\mathrm{Na}$ segunda parte, exploram-se as representações dos pesquisadores-orientadores do Provoc sobre os objetivos e as estratégias de orientação, à luz do referencial teórico escolhido com base nos dados empíricos. 
A visão dos pesquisadores-orientadores...

\subsection{O questionário}

Observa-se uma diferença significativa na quantidade de correlações entre os itens de uma mesma escala para cada dimensão, como mostra o Quadro 3.

\begin{tabular}{|c|c|c|}
\hline \multirow{2}{*}{ Escala } & \multicolumn{2}{|c|}{ Dimensões } \\
\cline { 2 - 3 } & Importância & Exeqüibilidade \\
\hline Objetivos (15 itens) & $\begin{array}{c}38 \text { correlações significativas entre } \\
\text { os itens }\end{array}$ & $\begin{array}{c}66 \text { correlações significativas } \\
\text { entre os itens }\end{array}$ \\
\hline Estratégias (10 itens) & $\begin{array}{c}14 \text { correlações significativas entre } \\
\text { os itens }\end{array}$ & $\begin{array}{c}42 \text { correlações significativas } \\
\text { entre os itens. }\end{array}$ \\
\hline
\end{tabular}

Quadro 3

Número de correlações significativas entre os itens de uma mesma escala para cada uma das dimensões

Este resultado corrobora as observações feitas sobre os níveis mais elevados de consistência interna na dimensão exeqüibilidade, em ambas as escalas. Os valores superiores obtidos nessa dimensão, tanto em termos de consistência interna quanto em termos de número de intercorrelações, sugerem que as representações dos pesquisadores sobre a I.C. expressamse no fazer. Assim, os objetivos e as estratégias transparecem no ato de orientar, pois se tornam tangíveis, viabilizando sua avaliação.

Além disso, observam-se diferenças nas correlações de um mesmo item em cada uma das dimensões. Por exemplo, na escala Objetivos, "incentivar o estudante a seguir a carreira científica" se correlaciona com "formar pesquisadores o mais precocemente possivep" e "incentivar o estudante a gostar de ciência e de suas atividades", na dimensão importância. No entanto, na dimensão exeqüibilidade, o item se correlaciona com "desenvolver no estudante a habilidade de solucionar problemas" e "fornecer ao estudante uma noção de problemas éticos da ciência".

Da mesma forma, na escala Estratégia, dimensão importância, "estimular o estudante a apresentar trabalhos cientificos" correlaciona-se apenas com o item "inseri-lo em um dos projetos de pesquisa em andamento, indicando um supervisor". No entanto, na dimensão exeqüibilidade, essa estratégia correlaciona-se com cinco itens:

- apresentar textos relacionados às pesquisas para que o estudante proponha um projeto;

- apresentar propostas de projetos investigativos que sejam apropriados ao seu nivel de formação;

- discutir com ele questões de investigação e procedimentos de pesquisa;

- discutir com o estudante a validade dos resultados obtidos;

- apresentar atividades que desenvolvam sua autoconfiança.

A estratégia "discutir com ele questões de investigação e procedimentos de pesquisa" é a que apresenta a maior diferença entre as duas dimensões no número de correlações: somente duas na dimensão importância e sete na dimensão exeqüibilidade. Essa informação aponta a dife- 
Filipecki, A. et al.

rença entre avaliar a importância de uma estratégia e a sua operacionalização, que dependeria de ações combinadas, processo bem mais complexo.

Números elevados de correlações entre os itens sugerem que estes são interdependentes, por exemplo, a importância em "contribuir para o desenvolvimento das condições pessoais do aluno" está associada à importância de 6 outros objetivos. Da mesma forma, a exeqüibilidade do objetivo "desenvolver no estudante a habilidade de solucionar problemas" está relacionada à exeqüibilidade de outros nove itens.

Quando são analisadas as correlações entre as escalas, na dimensão exeqüibilidade, observa-se que algumas estratégias se intercambiam com objetivos. Por exemplo, o objetivo original do Programa - "oportunizar a vivência do trabalho cientifico porparte dos estudantes" - pode ser entendido como uma estratégia que permite: "discutir com ele (estudante) questões de investigação e procedimentos de pesquisa" (20), "estimular o estudante a apresentar trabalhos científicos" (21), "treinar o estudante na utilização dos equipamentos e manipulação de amostras" (22), e "discutir com o estudante a validade dos resultados obtidos". Essa permuta entre objetivo e estratégia sugere uma revisão nos itens das escalas e uma nova redação do questionário.

Tendo em vista o número significativo de correlações na dimensão exeqüibilidade em ambas escalas, e também a diferença na natureza dessas correlações, foi dada ênfase à descrição das representações dos orientadores sobre a I.C. nesta dimensão, apresentada na próxima seção.

\subsection{As representações dos orientadores} sobre a I.C. de estudantes do Ensino Médio

A iniciação científica de estudantes de Ensino Médio, no Brasil, ainda é uma experiência nova. Portanto, seus objetivos estão sendo redefinidos constantemente. Além disso, não existe, na literatura, um número significativo de estudos que permita estabelecer a priori os referenciais a serem adotados. Utilizando-se os dados empíricos obtidos neste estudo e considerando-se a natureza multifacetada do processo de orientação científica - que envolve tanto aspectos não-formais de ensino-aprendizagem quanto questões relacionadas ao ambiente de pesquisa - foram selecionadas duas linhas teóricas: a teoria sociocognitiva de Bandura (2004) e a sociologia da ciência de Latour (2000).

A primeira permite compreender as mudanças psicossociais decorrentes da participação do orientando no Programa, mais especificamente quanto à interação entre orientadororientando e os demais atores envolvidos no processo de orientação. Os conceitos de "aprendizagem por imitação" e de "auto-eficácia" de Bandura se aplicam a um conjunto de objetivos e estratégias de orientação científica, posto que, segundo esse autor, condutas prescritas socialmente são, na sua maioria, aplicadas da mesma forma que foram aprendidas. Um exemplo seria a influência dos métodos didáticos dos mestres sobre seus alunos enquanto futuros professores. A aquisição de padrões de comportamento por tentativa e erro é menos eficiente e segura do que por observação do comportamento de outras pessoas. O estudante do Provoc observa uma variedade de modelos dentro do ambiente de pesquisa e nos eventos científicos dos quais participa. Essa informação codificada serve, posteriormente, como guia para a ação de novos comportamentos (Bandura apud Bahn, 2001). As estruturas mais complexas e os padrões inovativos, que não podem ser derivados apenas de experiências imitativas, são desenvolvidos com base nas estratégias de orientação combinadas à escolarização. 
As idéias de Latour permitem compreender em que medida a inserção do estudante nos laboratórios, vivenciando as práticas instituídas no seu interior, contribui para sua compreensão do processo de construção do conhecimento científico. Para o estudante de Ensino Médio, a pesquisa científica é uma espécie de "caixa-preta" que vai se abrindo ao longo do processo de orientação. Ao participar das diversas atividades de um laboratório que produz ciência, o estudante apreende que "fazer ciência" (Latour e Woolgar, 1997; Latour, 2000) é realmente um processo em construção que envolve verbas, força de trabalho, instrumentos, objetos novos, argumentos e inovações. A inserção do estudante no ambiente de pesquisa permite que o jovem conheça quem são as pessoas que estão fazendo ciência e como são distribuídas as responsablidades nesse espaço.

Assim, no sentido de compreender as representações dos pesquisadores sobre a I.C. de estudantes de ensino, na dimensão do fazer, os itens das escalas Objetivos e Estratégias da I.C. foram reagrupados em função do referencial adotado. Os objetivos foram organizados em 4 classes relacionadas aos seguintes aspectos: o ambiente físico, a aquisição de conhecimentos e habilidades, a carreira acadêmico-profissional e a mudança de atitude (Quadro 4). As estratégias (Quadro 5) foram distribuidas entre as classes: independência, acompanhamento e treinamento científico.

Uma visão compartilhada pelos pesquisadores-orientadores é o fato de que a investigação científica é uma "arte prática" (Ziman, 1979) que não se aprende nos livros, e sim por meio da imitação e da experiência. Sem o ambiente físico da pesquisa, materializado nos laboratórios e nos eventos científicos, não é possível desenvolver conhecimentos e habilidades inerentes à carreira científica. De fato, o aspecto cognitivo do Programa é o que apresenta maior ênfase para os pesquisadores, seguindo do aspecto atitudinal (aspectos sociocognitivos). Assim, a opção do estudante pela carreira científica estaria associada à sua crença de auto-eficácia. Isto é, na medida em que o orientando percebe sua capacidade para organizar e executar linhas de ação voltadas para a atividade científica e tem o domínio desta experiência, sente-se motivado para seguir a carreira científica. Além do sucesso (ou fracasso) fortalecer (ou enfraquecer) a crença de eficácia pessoal do orientando, a persuasão social do orientador e do grupo também desempenham papel preponderante nesse processo. De fato, as estratégias de orientação que levam em conta o nível de formação (item 19) e buscam desenvolver a autoconfiança do estudante (item 25) - estratégias que evitam colocar o orientando em situação prematura que o leve a fracassar - apresentam números elevados de correlação entre si e também com os objetivos.

A análise qualitativa dos dados coletados na terceira seção do questionário, conforme discutido na seção 3.1, confirma não apenas a importância atribuída pelo pesquisador à qualidade da orientação, como aponta sua influência "na decisão (do estudante) de seguir ou não o caminho da atividade cientifica". Observa-se ainda, nas falas de alguns pesquisadores, como a aprendizagem por imitação perpassa a convivência do estudante de Ensino Médio com estudantes de graduação e pós-graduação, contribuindo para a sua escolha profissional. De fato, a presença no laboratório de estudantes de diferentes níveis acadêmicos possibilita a utilização de uma estratégia de orientação denominada, entre os pesquisadores, de "filosofia do irmão mais velho": o estudante de pós-graduação auxilia o da graduação (PIBIC), e o do PIBIC auxilia o do Ensino Médio. 
Filipecki, A. et al.

Finalmente, não foram identificas diferenças significativas nos escores médios dos objetivos e das estratégias, em ambas as dimensões (importância e exeqüibilidade), em função dos anos de orientação e das áreas de conhecimento. Trata-se de um objeto que precisa ser mais explorado tendo em vista as limitações do próprio instrumento e o número de respondentes.

\begin{tabular}{|c|c|c|}
\hline Classes & Item & Objetivo \\
\hline \multirow{3}{*}{ Ambiente físico } & 8 & $\begin{array}{l}\text { Oportunizar a vivência do trabalho de laboratório } \\
\text { científico por parte dos estudantes. }\end{array}$ \\
\hline & 10 & $\begin{array}{l}\text { Permitir que o estudante conheça como os cientistas } \\
\text { trabalham profissionalmente. }\end{array}$ \\
\hline & 7 & $\begin{array}{l}\text { Possibilitar a participação de estudantes no } \\
\text { desenvolvimento de tarefas científicas. }\end{array}$ \\
\hline \multirow{4}{*}{$\begin{array}{l}\text { Conhecimentos e } \\
\text { habilidades }\end{array}$} & 3 & Auxiliar o estudante a pensar cientificamente. \\
\hline & 9 & $\begin{array}{l}\text { Desenvolver, no estudante, a habilidade de solucionar } \\
\text { problemas. }\end{array}$ \\
\hline & 11 & $\begin{array}{c}\text { Desenvolver, no estudante, a habilidade de integrar } \\
\text { teoria e prática. }\end{array}$ \\
\hline & 12 & $\begin{array}{l}\text { Oportunizar a aprendizagem de técnicas e } \\
\text { procedimentos de coleta e análise de dados. }\end{array}$ \\
\hline \multirow{4}{*}{$\begin{array}{l}\text { Carreira acadêmico- } \\
\text { profissional }\end{array}$} & 2 & Formar pesquisadores o mais precocemente possível. \\
\hline & 5 & Incentivar o estudante a seguir a carreira científica. \\
\hline & 6 & $\begin{array}{l}\text { Distinguir estudantes que se identifiquem com a } \\
\text { pesquisa científica. }\end{array}$ \\
\hline & 14 & $\begin{array}{l}\text { Auxiliar o estudante a fazer uma escolha profissional } \\
\text { mais consciente. }\end{array}$ \\
\hline \multirow[t]{4}{*}{ Atitudes } & 1 & $\begin{array}{c}\text { Despertar, nos estudantes do ensino médio, o interesse } \\
\text { pela pesquisa científica. }\end{array}$ \\
\hline & 4 & $\begin{array}{l}\text { Incentivar o estudante a gostar da ciência e de suas } \\
\text { atividades. }\end{array}$ \\
\hline & 13 & $\begin{array}{l}\text { Contribuir para o desenvolvimento das condições } \\
\text { pessoais do aluno. }\end{array}$ \\
\hline & 15 & $\begin{array}{l}\text { Fornecer ao estudante uma noção dos problemas } \\
\text { éticos da ciência. }\end{array}$ \\
\hline
\end{tabular}

Quadro 4

Agrupamento dos objetivos à luz do referencial adotado 
A visão dos pesquisadores-orientadores...

\begin{tabular}{|c|c|c|}
\hline Classes & Item & Estratégia \\
\hline \multirow{5}{*}{ Independência } & 17 & $\begin{array}{l}\text { Apresentar textos relacionados às pesquisas para que } \\
\text { o estudante proponha um projeto. }\end{array}$ \\
\hline & 19 & $\begin{array}{l}\text { Apresentar propostas de projetos investigativos que } \\
\text { sejam apropriados ao seu nível de formação. }\end{array}$ \\
\hline & 24 & $\begin{array}{l}\text { Fornecer modelos que possam auxiliá-lo no seu } \\
\text { desempenho profissional futuro. }\end{array}$ \\
\hline & 25 & $\begin{array}{l}\text { Apresentar atividades que desenvolvam sua } \\
\text { autoconfiança. }\end{array}$ \\
\hline & 21 & Estimular o estudante a apresentar trabalhos científicos. \\
\hline \multirow[t]{2}{*}{ Acompanhamento } & 16 & $\begin{array}{l}\text { Inseri-lo em um dos projetos de pesquisa em } \\
\text { andamento, indicando um supervisor. }\end{array}$ \\
\hline & 18 & $\begin{array}{l}\text { Avaliar a persistência do estudante na realização de } \\
\text { suas atividades }\end{array}$ \\
\hline \multirow{3}{*}{ Treinamento científico } & 20 & $\begin{array}{l}\text { Discutir com ele questões de investigação e } \\
\text { procedimentos de pesquisa. }\end{array}$ \\
\hline & 22 & $\begin{array}{l}\text { Treinar o estudante na utilização dos equipamentos e } \\
\text { manipulação de amostras. }\end{array}$ \\
\hline & 23 & $\begin{array}{l}\text { Discutir com o estudante a validade dos resultados } \\
\text { obtidos. }\end{array}$ \\
\hline
\end{tabular}

Quadro 5

Agrupamento das estratégias à luz do referencial adotado

\section{Conclusões}

Para a Coordenação do Provoc, a I.C. é uma atividade sistemática e disciplinadora, que utiliza instrumentos diferenciados de seleção e avaliação, na qual o estudante desenvolve atividades relacionadas ao planejamento, execução, interpretação e comunicação de uma investigação científica e dos seus resultados. Significa dizer que as análises apresentadas, neste estudo, são de caráter instigador.

Entende-se que as contribuições do Programa só podem ser compreendidas na medida em que cada uma das dimensões multifacetadas envolvidas no seu funcionamento venha a ser avaliada. Nesse sentido, questões relacionadas ao impacto da I.C. para estudantes de Ensino Médio continuam em aberto. Aspectos que ainda precisam ser mais estudados referem-se: à trajetória acadêmico-profissional dos orientandos; às relações entre o Programa e a escola de origem, principalmente os macrobenefícios do convênio instituição escolar - instituição de pesquisa e as formas de reconhecimento e recompensa profissional dos pesquisadores-orientadores.

Este trabalho aponta para um conjunto de objetivos que foram, ao longo dos anos, sendo adicionados aos objetivos originais do Provoc. Também mostra que, de modo geral, a 
Filipecki, A. et al.

exeqüibilidade dos objetivos e das estratégias descritas são inferiores à importância, o que reflete, em certa medida, a crença de auto-eficácia dos orientadores. Além disso, chama atenção o fato de os orientadores não atribuírem relevância significativa ao papel que desempenham enquanto modelo profissional futuro (item 25). Esse dado sugere a necessidade de se fazer um aprofundamento das questões relacionadas ao papel do pesquisador como modelo (role play).

Considerando a faixa etária desses estudantes, as condições atuais da escola e os objetivos e as estratégias de orientação científica analisados neste trabalho, observa-se que a I.C. demanda do pesquisador uma abordagem "quase-artesanal", o que limita o número de alunos que podes ser atendidos pelo Provoc.

Seria importante, ainda, desenvolver uma metodologia que permitisse avaliar em que medida os objetivos relacionados ao pensamento científico - integrar teoria e prática, solucionar problemas, sistematizar procedimentos no laboratório, ter visão da ciência etc. - são alcançados pelo estudante, principalmente aqueles que convivem no ambiente de pesquisa durante os três anos das etapas da I.C. Iniciação e Avançado.

\section{Referências}

BAHN, D. Social learning theory: its application in the context of nurse education. Nurse Education Today, v. 21, p. 110-117, 2001.

BANDURA, A. Swimming against the mainstream: the early years from chilly tributary to transformative mainstream. Behaviour Research and Therapy, v. 42, p. 613-630, 2004.

BARDIN, L. Análise de conteúdo. Lisboa: Edições 70, 1977.

BZUNECK, J. A. As crenças de auto-eficácia e o seu papel na motivação do aluno. In: BORUCHOVITCH, E.; BZUNECK, J. A (Org.). A motivação do aluno: contribuições da psicologia contemporânea. Petrópolis: Vozes, s/d. p. 116-133.

DIMITRIS, P.; NIEDDERER, H. Case studies on lab work on five european countries working paper. 1998. Disponível em: <http://www.education.leeds.ac.uk/research/scienceed/labwork.htm>, 1998.

FILIPECKI, A. Representações sociais do pesquisador-orientador sobre o processo de trabalho de orientação científica de jovens de Ensino Médio. Relatório de Pesquisa. Rio de Janeiro, 2004.

GANE, C.; SARSON, T. Análise estruturada de sistemas. Rio de Janeiro: LTC, 1995. 
A visão dos pesquisadores-orientadores...

JORNAL VOX POLI No ${ }^{\circ}$ 6. Rio de Janeiro: Escola Politécnica da Saúde Joaquim Venâncio, 1998.

KERLINGER, F. Metodologia da pesquisa em ciências sociais: um tratamento conceitual. São Paulo: EPU, 1980.

LATOUR, B. Ciência em ação: como seguir cientistas e engenheiros sociedade afora. São Paulo: UNESP, 2000.

LATOUR, B.; WOOLGAR, S. A vida de laboratório: a produção dos fatos científicos. Rio de Janeiro: Relume Dumará, 1997.

ZIMAN, J. O homem e a ciência: conhecimento público. São Paulo: Editora da Universidade de São Paulo, 1979.

Artigo recebido em dezembro de 2005 e aceito em julho de 2006. 